\title{
Invertible Darboux Transformations ${ }^{\star}$
}

\author{
Ekaterina SHEMYAKOVA
}

Department of Mathematics, SUNY at New Paltz, 1 Hawk Dr. New Paltz, NY 12561, USA

E-mail: shemyake@newpaltz.edu

Received October 01, 2012, in final form January 01, 2013; Published online January 04, 2013

http://dx.doi.org/10.3842/SIGMA.2013.002

\begin{abstract}
For operators of many different kinds it has been proved that (generalized) Darboux transformations can be built using so called Wronskian formulae. Such Darboux transformations are not invertible in the sense that the corresponding mappings of the operator kernels are not invertible. The only known invertible ones were Laplace transformations (and their compositions), which are special cases of Darboux transformations for hyperbolic bivariate operators of order 2. In the present paper we find a criteria for a bivariate linear partial differential operator of an arbitrary order $d$ to have an invertible Darboux transformation. We show that Wronkian formulae may fail in some cases, and find sufficient conditions for such formulae to work.
\end{abstract}

Key words: Darboux transformations; Laplace transformations; 2D Schrödinger operator; invertible Darboux transformations

2010 Mathematics Subject Classification: 37K10; 37K15

\section{Introduction}

Darboux transformations are shape preserving transformations of linear partial differential operators. These transformations were originally introduced in [2] first for operators

$$
-D_{x}^{2}+u-\lambda
$$

corresponding to Sturm-Liouville equations, where $u \in K$, and where $K$ is some differential field (see Section 2), and $\lambda$ is a constant, and, secondly, for operators of the form

$$
D_{x} D_{y}+a D_{x}+b D_{y}+c,
$$

where $a, b, c \in K$, which is a form equivalent up to a change of variable to stationary 2D Schrödinger operator. These transformations were suggested as a possible method for the solution of the corresponding linear partial differential equations ${ }^{1}$. By exploiting these ideas many new kinds of integrable 1D and 2D Schrödinger equations have been discovered. See, for example, this influential paper [8].

Decades later, Darboux transformations became a standard tool of the inverse scattering method, where they are applied to the linear partial differential operators forming the Lax pair and thus eventually serve for the solution of nonlinear partial differential equations $[6,9]$.

A Darboux transformation for an arbitrary linear partial differential operator $\mathbf{L}$ can be defined as follows. An operator $\mathbf{L}$ is transformed into operator $\mathbf{L}_{1}$ with the same principal symbol (see Section 2) by means of operator $\mathbf{M}$ if there is a linear partial differential operator $\mathbf{N}$ such that

$$
\mathbf{N L}=\mathbf{L}_{1} \mathbf{M}
$$

${ }^{\star}$ This paper is a contribution to the Special Issue "Symmetries of Differential Equations: Frames, Invariants and Applications". The full collection is available at http://www.emis.de/journals/SIGMA/SDE2012.html

${ }^{1}$ Though we cannot guarantee in advance that it will be successful. 
In this case we shall say that there is a Darboux transformation for pair $(\mathbf{L}, \mathbf{M})$; we also say that $\mathbf{L}$ admits a Darboux transformation generated by $\mathbf{M}$. We define the order of a Darboux transformation as the order of the $\mathbf{M}$ corresponding to it.

If a linear partial differential operator $\mathbf{L} \in K\left[D_{x}, D_{y}\right]$ has a right factor $D_{x}+l$, then it admits a Darboux transformation generated by $\mathbf{M}=D_{x}+l$. Indeed, suppose $\mathbf{L}=\mathbf{F}\left(D_{x}+l\right)$, then for arbitrary $t \in K:\left(D_{x}+t\right) \mathbf{L}=\left(D_{x}+t\right) \mathbf{F}\left(D_{x}+l\right)$, that is $\mathbf{L}$ transforms into $\left(D_{x}+t\right) \mathbf{F}$. However, a general Darboux transformation is not implied by a factorization.

For operator (1) the only possible Darboux transformations are those generated by $\mathbf{M}=D_{x}-$ $\psi_{1, x} \psi_{1}^{-1}$, where $\psi_{1}$ is in the kernel of the operator (1). For operator (2) Darboux transformations can be generated by each of the following:

$$
\begin{aligned}
& \mathbf{M}_{y}=D_{x}-\psi_{1, x} \psi_{1}^{-1}, \\
& \mathbf{M}_{x}=D_{y}-\psi_{1, y} \psi_{1}^{-1},
\end{aligned}
$$

where $\psi_{1}$ is in the kernel of operator (2). For operator (2), however, there are also Darboux transformations that are generated by $\mathbf{M}$ of a form $D_{x}+m$ or $D_{y}+m, m \in K$, but cannot be represented as either (4) or (5). These transformations are known as Laplace transformations. Specifically, these transformations are transformations of operators $\mathbf{L}$ of the form (2) and are generated by $\mathbf{M}=D_{x}+b$, or $\mathbf{M}=D_{y}+a$, provided the Laplace invariant $k=b_{y}+a b-c$ is not zero (or the invariant $h=a_{x}+a b-c$ is not zero). See more about Laplace transformations in [12]. It is also known that Laplace transformations are invertible [3].

Moreover, it has been proved [10] that Laplace transformations are the only two Darboux transformations of (2) generated by $\mathbf{M}$ of a form $D_{x}+m$ or $D_{y}+m, m \in K$ that cannot be represented as either (4) or (5). Operators (4) and (5) can be equivalently defined by their action on functions in terms of Wronskians:

$$
\begin{aligned}
& \mathbf{M}_{x}(\psi)=\left(D_{x}-\frac{\psi_{1, x}}{\psi_{1}}\right)(\psi)=\frac{\left|\begin{array}{cc}
\psi & \partial_{x} \psi \\
\psi_{1} & \partial_{x} \psi_{1}
\end{array}\right|}{-\left|\psi_{1}\right|}, \\
& \mathbf{M}_{y}(\psi)=\left(D_{y}-\frac{\psi_{1, y}}{\psi_{1}}\right)(\psi)=\frac{\left|\begin{array}{cc}
\psi & \partial_{y} \psi \\
\psi_{1} & \partial_{y} \psi_{1}
\end{array}\right|}{-\left|\psi_{1}\right|} .
\end{aligned}
$$

The consecutive application of a sequence of $n$ Darboux transformations leads to one Darboux transformation of order $n$, which then can be defined in terms of Wronskians of order $n$. Indeed, if we define a $(t, s)$ Wronkian, $t+s=n-1$, of functions $f_{1}, \ldots, f_{t+s}$ as the $n \times n$ determinant

$$
W_{t, s}\left(f, f_{1}, \ldots, f_{t+s}\right)=\left|\begin{array}{ccccccc}
f & \partial_{x} f & \ldots & \partial_{x}^{t} f & \partial_{y} f & \ldots & \partial_{y}^{s} f \\
\ldots & \ldots & \ldots & \ldots & \ldots & \ldots & \ldots \\
f_{t+s} & \partial_{x} f_{t+s} & \ldots & \partial_{x}^{t} f_{t+s} & \partial_{y} f_{t+s} & \ldots & \partial_{y}^{s} f_{t+s}
\end{array}\right|,
$$

then we can reformulate Darboux's original result [2] and join it to the result [13] for operators of the form

$$
D_{x} D_{x}+a D_{x}+b D_{y}+c, \quad a, b, c \in K
$$

as follows.

Theorem 1. If $m+n$ linearly independent $\psi_{1}, \ldots, \psi_{m+n}$ in the kernel of $\mathbf{L}$ of the form (2) or (6) are given, then operator $\mathbf{M}$ given by

$$
\mathbf{M}(\psi)=(-1)^{n+m} \frac{W_{m, n}\left(\psi, \psi_{1}, \ldots, \psi_{m+n}\right)}{W_{m-1, n}\left(\psi_{1}, \ldots, \psi_{m+n}\right)}
$$

defines some Darboux transformation for $\mathbf{L}$. 
In [1], which preceded [13], this theorem was proved for operators of the form (6) with constant coefficients. The general question whether a Darboux transformation of order $n$ can be represented as a sequence of Darboux transformations of order one is open for operators of the form (2). In [11] the statement was proved for operators of the form (2) with arbitrary (i.e. not necessarily constant) coefficients, but for transformations of order two only. For operators of the form (1), the statement is an implication of the Crum theorem [6], which states that a Darboux transformation of order $n$ is generated by $\mathbf{M}$ which can be defined by the same Wronskian formulae (with the only difference that this is a case of a single variable):

$$
\mathbf{M} \psi=\frac{W_{n-1,0}\left(\psi_{1}, \ldots, \psi_{n}, \psi\right)}{W_{n-2,0}\left(\psi_{1}, \ldots, \psi_{n}\right)} .
$$

Generalizations of Darboux transformations have been introduced for other types of operators and for systems of operators. Thus, in [5] one is defined in terms of a twisted derivation $D$ satisfying $D(A B)=D(A)+\sigma(A) B$, where $\sigma$ is a homomorphism. Such twisted derivations include regular derivations, difference and q-difference operators and superderivatives as special cases. The corresponding $\mathbf{M}$ can be expressed by the same formulae in terms of Wronskians.

However, a straightforward generalization of Theorem 1 would not be true for many other kinds of operators. This fact has not been much stressed in relevant papers. Below is possibly the first explicit example of such a situation.

Example 1. Consider operator $\mathbf{L}=D_{x}^{2} D_{y}+y D_{x}^{2}+x D_{y}^{2}+1$ and an element of its kernel,

$$
\psi_{1}=\sin \left(\frac{y}{\sqrt{x}}\right) \in \operatorname{ker} \mathbf{L} .
$$

Straightforward computations show that no $\mathbf{M}$ in the form (4), nor in the form (5), generates a Darboux transformation for operator $\mathbf{L}$. There are, however, other $\psi_{1} \in$ ker $\mathbf{L}$, which generate Darboux transformations with $\mathbf{M}$ in the form (4) or (5).

In the present paper we are interested in answering the following questions.

1. Laplace transformations are known to be invertible, but essentially no other invertible transformations are known. Are there such transformations? Can we study their nature?

2. Example 1 indicates that Wronkian formulae do not work in some cases. Can we describe cases in which Wronkian formulae do work?

Specifically, in the present paper we show that there are many more kinds of operators than just the one (2) which admit invertible (see the precise definition in Section 4) Darboux transformations. We give criteria which allow us to describe all possible invertible Darboux transformations of arbitrary linear partial differential operators $\mathbf{L}$ and generated by $\mathbf{M}$ in the form $D_{x}+m$ or $D_{y}+m$. Given an arbitrary operator $\mathbf{L} \in K\left[D_{x}, D_{y}\right]$, we give sufficient conditions that guarantee that Wronskian formulae (4) and (5) work.

This paper is organized as follows. Section 2 outlines notation. Section 3 starts with the simple fact that a Darboux transformation for a pair $\left(\mathbf{L}, \mathbf{M}=D_{x}+m\right), m \in K$ exists if and only if a Darboux transformation for pair $\left(\mathbf{L}^{g}, D_{x}\right.$ ) exists (analogously, for pairs with $\mathbf{M}=D_{y}+m$ ). Then Theorem 2 states necessary and sufficient conditions that guarantee that a Darboux transformation for pair $\left(\mathbf{L}^{g}, D_{x}\right)$ exists. In Section 4 we define invertible transformations as transformations such that the corresponding mapping $\operatorname{ker} \mathbf{L} \rightarrow \operatorname{ker} \mathbf{L}_{1}$ is invertible. Theorem 3 states necessary and sufficient conditions that guarantee that a Darboux transformation for pair $\left(\mathbf{L}^{g}, D_{x}\right)$ is invertible (as well as for pairs with $\mathbf{M}=D_{y}+m$ ). It also describes all other possible cases for the dimension of the kernel of mapping $\operatorname{ker} \mathbf{L} \rightarrow \operatorname{ker} \mathbf{L}_{1}$. In Section 5 Theorem 4 states 
that if in the kernel of operator $\mathbf{L}$ there is a subspace of large enough dimension generated by elements that differ from each other by a multiplication of a function of variable $y$, then each of those elements $\psi_{1}$ gives the same $\mathbf{M}=D_{x}-\psi_{1, x} \psi_{1}^{-1}$, and this $\mathbf{M}$ generates a Darboux transformation. In other words, we state when Darboux Wronskian formulae work for arbitrary bivariate linear partial differential operator. There is also an analogous statement for the case $\mathbf{M}=D_{y}-\psi_{1, y} \psi_{1}^{-1}$.

\section{Preliminaries}

Let $K$ be a differential field of characteristic zero with commuting derivations $\partial_{x}, \partial_{y}$. Let $K\left[D_{x}, D_{y}\right]$ be the corresponding ring of linear partial differential operators over $K$, where $D_{x}, D_{y}$ correspond to derivations $\partial_{x}, \partial_{y}$.

Operators $\mathbf{L} \in K\left[D_{x}, D_{y}\right]$ have the general form

$$
\mathbf{L}=\sum_{i+j=0}^{d} a_{i j} D_{x}^{i} D_{y}^{j}, \quad a_{i j} \in K .
$$

The formal polynomial

$$
\operatorname{Sym}_{\mathbf{L}}=\sum_{i+j=d} a_{i j} X^{i} Y^{j}
$$

in the formal variables $X, Y$ is called the principal symbol of $\mathbf{L}$.

One can either assume the field $K$ to be differentially closed, in other words containing all the solutions of, in general nonlinear, partial differential equations with coefficients in $K$, or simply assume that $K$ contains the solutions of those partial differential equations that we encounter on the way.

Let $f \in K$, and $\mathbf{L} \in K\left[D_{x}, D_{y}\right]$; by $\mathbf{L} f$ we denote the composition of operator $\mathbf{L}$ with the operator of multiplication by a function $f$, while $\mathbf{L}(f)$ mean the application of operator $\mathbf{L}$ to $f$. For example,

$$
D_{x} f=f D_{x}+f_{x} \in K\left[D_{x}, D_{y}\right], \quad D_{x}(f)=f_{x} \in K .
$$

The second lower index attached to a symbol denoting a function means the derivative of that function with respect to the variables listed there. For example,

$$
f_{1, x y y}=\partial_{x} \partial_{x} \partial_{y} f_{1} .
$$

Definition 1. Given some operator $\mathbf{R} \in K\left[D_{x}, D_{y}\right]$ and an invertible function $g \in K$, the corresponding gauge transformation is defined as

$$
\mathbf{R} \rightarrow \mathbf{R}^{g}, \quad \mathbf{R}^{g}=g^{-1} \circ R \circ g,
$$

where $\circ$ denotes the operation of the composition of operators in $K\left[D_{x}, D_{y}\right]$.

Remark 1. The principal symbol of an operator in $K\left[D_{x}, D_{y}\right]$ is invariant under the gauge transformations.

Lemma 1. Let $\mathbf{M}, \mathbf{L} \in K\left[D_{x}, D_{y}\right]$ and let a Darboux transformation exist for the pair $(\mathbf{M}, \mathbf{L})$. Then one exists also for $\left(\mathbf{M}^{g}, \mathbf{L}^{g}\right)$, where $g$ is an arbitrary invertible element of $K$.

Proof. Indeed, (3) implies $g^{-1} \circ \mathbf{N} \circ g \circ g^{-1} \circ \mathbf{L} \circ g=g^{-1} \circ \mathbf{L}_{1} \circ g \circ g^{-1} \circ \mathbf{M} \circ g$, and, therefore, $\mathbf{N}^{g} \circ \mathbf{L}^{g}=\mathbf{L}_{1}^{g} \circ \mathbf{M}^{g}$. Since gauge transformations do not change the symbol of an operator, the proof is complete. 


\section{Darboux transformations generated by $\mathrm{M}=D_{x}$ or $\mathrm{M}=D_{y}$}

Lemma 2. Let $\mathbf{L}$ be an arbitrary linear partial differential operator in $K\left[D_{x}, D_{y}\right]$. If there exists a Darboux transformation for the pair $\left(\mathbf{L}, \mathbf{M}=D_{x}+m\right), m \in K$ then there is a Darboux transformation for pair $\left(\mathbf{L}^{g}, D_{x}\right)$. If there exists a Darboux transformation for pair $(\mathbf{L}, \mathbf{M}=$ $\left.D_{y}+m\right)$, then there exists a Darboux transformation for pair $\left(\mathbf{L}^{g}, D_{y}\right)$.

Proof. Consider a Darboux transformation for the pair $\left(\mathbf{L}, \mathbf{M}=D_{x}+m\right)$, and consider $g$, a solution of $g_{x} / g=-m$. Then $\mathbf{M}^{g}=D_{x}$. Then by Lemma 1 there is also a Darboux transformation for pair $\left(\mathbf{L}^{g}, D_{x}\right)$. Analogous reasoning applies for the pair $\left(\mathbf{L}, \mathbf{M}=D_{y}+m\right)$.

Theorem 2. Let $\mathbf{L}$ be an arbitrary linear partial differential operator in $K\left[D_{x}, D_{y}\right]$, that is, an operator of the form (7).

Operator $\mathbf{L}$ has a Darboux transformation generated by $\mathbf{M}=D_{x}$ if and only if

$$
a_{0 j}=G_{j k}(y) a_{0 k}
$$

for all non-zero $a_{0 j}$ and $a_{0 k}, j=0, \ldots, d, k=0, \ldots, d$. Here $G_{j k}(y)$ are some functions of the variable $y$.

Operator $\mathbf{L}$ has a Darboux transformation generated by $\mathbf{M}=D_{y}$ if and only if

$$
a_{i 0}=F_{i k}(x) a_{k 0}
$$

for all non-zero $a_{i 0}$ and $a_{k 0}, i=0, \ldots, d, k=0, \ldots, d$. Here $F_{i k}(x)$ are some functions of the variable $x$.

Proof. Let there be a Darboux transformation for the pair $\left(\mathbf{L}, D_{x}\right)$. This means that for some $\mathbf{L}_{1} \in K\left[D_{x}, D_{y}\right]$ and $n \in K$

$$
\left(D_{x}+n\right) \mathbf{L}=\mathbf{L}_{1} D_{x} .
$$

The right hand side of this operator equality does not contain terms of the form $c D_{y}^{j}, c \in K$. On the other hand for $j=1, \ldots, d$ the coefficient of $D_{y}^{j}$ in the left hand side is

$$
n a_{0 j}+a_{0 j, x}=0 .
$$

The 'free' coefficient on the right hand side of (10) is zero, while on the left hand side it is

$$
n a_{00}+a_{00, x}=0 .
$$

Equating the expressions for $n$ obtained from each of the equalities (11) and (12), we see that condition (8) is satisfied.

The analogous statement for the case $\mathbf{M}=D_{y}$ can be proved similarly.

To prove the theorem in the other direction, suppose that the conditions (8) are satisfied. Consider then the operator equality (10) defining a Darboux transformation for the pair $\left(\mathbf{L}, D_{x}\right)$. It implies equalities (11), (12), from which $n$ can be determined uniquely and without contradiction. In general, equality (10), which is an equality of operators of orders $d+1$, implies $(d+3)(d+2) / 2$ equalities of the corresponding coefficients, of which $(d+1)$ are equalities $(11)$ and (12). Thus, we have a linear algebraic system of a maximum $(d+3)(d+2) / 2-(d+1)$ equations to solve. The unknowns in this system are the coefficients of $\mathbf{L}_{1}$, an operator of order $d$ and their number is $(d+2)(d+1) / 2$. Thus the number of equations in this system is less than or equal to the number of unknowns, so there is at least one non-zero solution.

Analogously we can prove that conditions (9) guarantee that there is a Darboux transformation for pair $\left(\mathbf{L}, D_{y}\right)$. 


\section{Invertible Darboux transformations}

Consider a Darboux transformation $\mathbf{L} \rightarrow \mathbf{L}_{1}$ of operators in $K\left[D_{x}, D_{y}\right]$ generated by an operator $\mathbf{M} \in K\left[D_{x}, D_{y}\right]$. This transformation implies a mapping of the linear vector spaces

$$
\operatorname{ker} \mathbf{L} \rightarrow \operatorname{ker} \mathbf{L}_{1}: \quad w \mapsto \mathbf{M}(w) .
$$

This mapping is invertible if it is an isomorphism, that is if its kernel is zero

$$
\operatorname{ker} \mathbf{L} \cap \operatorname{ker} \mathbf{M}=\{0\} \text {. }
$$

In this case we shall say that the Darboux transformation is invertible.

Remark 2. Darboux transformations of the operator $\mathbf{L} \in K\left[D_{x}, D_{y}\right]$ generated by $\mathbf{M}$ constructed using Wronkian formulae are not invertible. Indeed, we take some number of known solutions of $\mathbf{L}(\psi)=0$ and then construct $\mathbf{M}$ as a Wronkian. Then by construction all those solutions belong to the kernel of $\mathbf{M}$.

Theorem 3. If the operator $\mathbf{L} \in K\left[D_{x}, D_{y}\right]$ given by (7) admits a Darboux transformation generated by $\mathbf{M}=D_{x}$ or $\mathbf{M}=D_{y}$ then

$$
\operatorname{dim}(\operatorname{ker}(\mathbf{L}) \cap \operatorname{ker}(\mathbf{M}))= \begin{cases}\infty, & a_{0 k}=0, \quad \forall k \in\{0, \ldots, d\}, \\ d_{y}, & \text { otherwise }\end{cases}
$$

where $d_{y}$ is the largest $j$ such that $a_{0 j} \neq 0$. In particular, we have a criteria for a Darboux transformation generated by $\mathbf{M}=D_{x}$ or $\mathbf{M}=D_{y}$ to be invertible. Namely, it is invertible if and only if all of the following hold

$$
a_{0 k}=0 \quad \forall k \in\{1, \ldots, d\}, \quad a_{00} \neq 0 .
$$

Proof. Suppose there is a Darboux transformation for the pair $\left(\mathbf{L}, D_{x}\right)$. The kernel of the operator $\mathbf{M}$ consists of functions of the form $g=g(y)$.

Let there be index $k, k>0$ such that the coefficients of $D_{y}^{k}, k=1, \ldots, d$ are not zero: $a_{0 k} \neq 0$. Then using (8) compute

$$
\mathbf{L}(g(y))=\sum_{j=0}^{d} a_{0 j} D_{y}^{j}(g(y))=a_{0 k} \sum_{j=0}^{d} G_{j k}(y) D_{y}^{j}(g(y)) .
$$

Thus, $\mathbf{L}(g(y))=0$ is equivalent to a linear differential equation of order $d_{y}$. The solution space of such an equation has dimension $d_{y}$.

If there is no such $k$ that the coefficient at $D_{y}^{k}, k=1, \ldots, d$ is not zero, then either $a_{00}=0$ and then $\operatorname{ker} \mathbf{L} \cap \operatorname{ker} \mathbf{M}=\{f(y)\}$, or $a_{00} \neq 0$ and $\operatorname{ker} \mathbf{L} \cap \operatorname{ker} \mathbf{M}=\{0\}$.

The statement for the pair $\left(\mathbf{L}, D_{y}\right)$ can be proved analogously.

Corollary 1. Laplace transformations (see Section 1) are the only invertible Darboux transformation generated by $\mathbf{M}=D_{x}+m$ or by $\mathbf{M}=D_{y}+m$ for operators in the form $D_{x} D_{y}+a D_{x}+$ $b D_{y}+c, a, b, c \in K$.

Proof. In [10] it has been proved that a Darboux transformation generated by $\mathbf{M}=D_{x}+m$ or by $\mathbf{M}=D_{y}+m$ is either a Laplace transformation, that is generated by $\mathbf{M}=D_{x}+b$ or $\mathbf{M}=D_{y}+a$, or generated by $\mathbf{M}$ in the form $D_{x}-\psi_{1, x} \psi_{1}^{-1}$, or $D_{y}-\psi_{1, y} \psi_{1}^{-1}$, where $\psi_{1} \in \operatorname{ker} \mathbf{L}$. In the latter case, $\psi_{1} \in(\operatorname{ker} \mathbf{L} \cap \operatorname{ker} \mathbf{M})$, and, therefore, the mapping $\operatorname{ker} \mathbf{L} \rightarrow \operatorname{ker} \mathbf{L}_{1}$ is not invertible. 
Consider a Darboux transformation for pair $\left(\mathbf{L}, \mathbf{M}=D_{x}+b\right)$. To use the criteria obtained in Theorem 3, we consider a gauge transformation of both operators of this pair. We use $g \in K$ such that $\mathbf{M}$ becomes $D_{x}$, that is $\mathbf{M}^{g}=D_{x}$. Such $g$ can be easily found as any solution of $g_{x} g^{-1}=-b$. Consider now $\mathbf{L}^{g}$,

$$
\begin{aligned}
\mathbf{L}^{g} & =D_{x} D_{y}+\left(a+g_{y} g^{-1}\right) D_{x}+\left(b+g_{x} g^{-1}\right) D_{y}+c+a g_{x} g^{-1}+b g_{y} g^{-1}+g_{x y} g^{-1} \\
& =D_{x} D_{y}+\left(a+g_{y} g^{-1}\right) D_{x}+c-a b+b g_{y} g^{-1}+g_{x y} g^{-1} \\
& =D_{x} D_{y}+\left(a+g_{y} g^{-1}\right) D_{x}+c-a b-b_{y} \\
& =D_{x} D_{y}+\left(a+g_{y} g^{-1}\right) D_{x}+k,
\end{aligned}
$$

where $k$ is one of two Laplace invariants of $\mathbf{L}$ (see Section 1 ). Thus, $k \neq 0$ is the necessary and sufficient condition for the Darboux transformation for the pair $\left(\mathbf{L}, \mathbf{M}=D_{x}+b\right)$ to be invertible. Analogously, $h \neq 0$ is the necessary and sufficient condition for Darboux transformation for pair $\left(\mathbf{L}, \mathbf{M}=D_{y}+a\right)$ to be invertible.

Example 2 (an invertible Darboux transformation). Consider the operator

$$
\mathbf{L}=D_{x} D_{y}^{2}+D_{x}^{2}+x D_{x}+1 .
$$

The coefficients of $\mathbf{L}$ satisfy condition (8), and, therefore, there is a Darboux transformation for $\mathbf{L}$ generated by $\mathbf{M}=D_{x}$. Since there is no term in the form $D_{y}^{i}$ and $\mathbf{L}(1)=1 \neq 0$, then by Theorem 3 the Darboux transformation for pair $(\mathbf{L}, \mathbf{M})$ is invertible. This Darboux transformation takes $\mathbf{L}$ into

$$
\mathbf{L}_{1}=D_{x} D_{y}^{2}+D_{x}^{2}+x D_{x}+2
$$

The corresponding operator $\mathbf{N}$ is $D_{x}$. In this case there is an inverse transformation of operators, $\mathbf{L}_{1} \mapsto \mathbf{L}$ by means of $\mathbf{M}^{\prime}=\mathbf{N}^{\prime}=D_{x}$.

From any such example one can generate a whole series of examples of Darboux transformations with $\mathbf{M}$ of the form $D_{x}+m$ or $D_{y}+m, m \neq 0$ by considering gauge transformations of $\mathbf{L}$ and $\mathbf{M}$.

\section{Solution-based Darboux transformations}

Theorem 4. Let an operator $\mathbf{L}$ be of the form $\mathbf{L}=\sum_{i+j=0}^{d} a_{i j} D_{x}^{i} D_{y}^{j}, a_{i j} \in K$, and $k=d$ if $a_{0 d} \neq 0$, and $k=d-1$, otherwise. Then if there are

$$
\psi_{1}, \psi_{2}, \ldots, \psi_{k} \in \operatorname{ker} \mathbf{L} /\{0\}
$$

such that

$$
\frac{\psi_{i}}{\psi_{1}}=T_{i}(y)
$$

for some non-constant functions $T_{i}(y) \in K, i=2, \ldots, k$, and

$$
W_{0, k-1}\left(1, T_{2}, \ldots, T_{k}\right) \neq 0,
$$

then there is a Darboux transformation for $\mathbf{L}$ generated by

$$
\mathbf{M}=D_{x}-\frac{\psi_{1, x}}{\psi_{1}} .
$$

Analogously, if instead of (13) and (14) conditions $\psi_{i} \psi_{1}^{-1}=F_{i}(x)$ and $W_{k-1,0}\left(1, T_{2}, \ldots, T_{k}\right) \neq 0$ hold, then there is a Darboux transformation for $\mathbf{L}$ generated by $\mathbf{M}=D_{y}-\psi_{1, y} \psi_{1}^{-1}$. 
Remark 3. Condition (14) implies that $\psi_{1}, \psi_{2}, \ldots, \psi_{k}$ are linearly independent. The condition that $\psi_{1}, \psi_{2}, \ldots, \psi_{k}$ are linearly independent does not necessarily imply condition (14).

Proof. Assume we have non-zero $\psi_{1}, \psi_{2}, \ldots, \psi_{k} \in \operatorname{ker} \mathbf{L}$, such that (13) and (14) hold. Then for the operator $\mathbf{L}^{\prime}=\mathbf{L}^{\psi_{1}}=\sum_{i+j=0}^{d} a_{i j}^{\prime} D_{x}^{i} D_{y}^{j}, a_{i j}^{\prime} \in K$ we have

$$
1 \in \operatorname{ker} \mathbf{L}^{\prime}, \quad T_{i}(y) \in \operatorname{ker} \mathbf{L}^{\prime}, \quad i=1, \ldots, k .
$$

The former means that $a_{00}^{\prime}=0$, while the latter implies that

$$
\sum_{j=1}^{d} a_{0 j}^{\prime} D_{y}^{j}\left(T_{i}(y)\right)=0, \quad i=2, \ldots, k
$$

which is a linear system of $k-1$ equations. The number of unknowns $a_{0 j}^{\prime}$ is the number of non-zero $a_{0 j}^{\prime}, j=1, \ldots, d$. If $k=d$, then the number of the unknowns is less or equal to $k$. If $k=d-1$, then $a_{0 d}=0$, and since the principal symbol of an operator is invariant with respect to the gauge transformations, $a_{0 d}^{\prime}=a_{0 d}=0$. Which means that in this case the number of unknowns is also less than or equal to $k$.

The Wronkian in (14) has the first column $(1,0, \ldots, 0)^{t}$, and the first row $\left(1, T_{2}, \ldots T_{k}\right)$. Thus, if we remove the first column and the first row, the rank of the corresponding matrix is $k-1$. Then the rank of the transpose of the latter matrix is also $k-1$,

$$
\operatorname{rank}\left(\begin{array}{ccc}
T_{2}^{\prime} & \ldots & T_{2}^{(k-1)} \\
\ldots & \ldots & \ldots \\
T_{k}^{\prime} & \ldots & T_{k}^{(k-1)}
\end{array}\right)=k-1 .
$$

This is a $(k-1) \times(k-1)$ matrix and adding extra column $\left(T_{2}^{k}, \ldots, T_{k}^{k}\right)^{t}$ on the right does not change the rank. Thus linear system (15) has matrix with rank $k-1$ and less or equal to $k$ unknowns, and, therefore, condition

$$
a_{0 j}^{\prime}=G_{j k}(y) a_{0 k}^{\prime}
$$

holds for all non-zero $a_{0 j}^{\prime}$ and $a_{0 k}^{\prime}, j=0, \ldots, d, k=0, \ldots, d$ for some $G_{j k}(y) \in K$. Thus, by Theorem 2 there exists a Darboux transformation for the pair $\left(\mathbf{L}^{\prime}, D_{x}\right)$. Therefore, there is a Darboux transformation for pair $\left(\mathbf{L}, \psi_{1} D_{x} \psi_{1}^{-1}\right)$, that is for pair $\left(\mathbf{L}, D_{x}-\psi_{1, x} \psi_{1}^{-1}\right)$.

The second statement, giving sufficient conditions for the existence of a Darboux transformation for $\mathbf{L}$ generated by $\mathbf{M}=D_{y}-\psi_{1, y} \psi_{1}^{-1}$, can be proved analogously.

Example 3. For operators of the form $D_{x} D_{y}+a D_{x}+b D_{y}+c, a, b, c \in K k=1$, Theorem 4 then means that it is enough to have one $\psi \in \operatorname{ker} \mathbf{L}$ to guarantee that a Darboux transformation for pair $\left(\mathbf{L}, \mathbf{M}=D_{x}-\psi_{1, x} \psi_{1}^{-1}\right)$ exists. This agrees with result of Theorem 1 .

Remark 4. The statement of Theorem 4 is not true when formulated in the opposite direction. Let there be a Darboux transformation for the pair $\left(\mathbf{L}, \mathbf{M}=D_{x}-\psi_{1, x} \psi_{1}^{-1}\right)$, where $\psi_{1} \in \operatorname{ker} \mathbf{L}$. Then there is a Darboux transformation for the pair $\left(\mathbf{L}^{\prime}=\mathbf{L}^{\psi_{1}}, \mathbf{M}^{\prime}=\mathbf{M}^{\psi_{1}}=D_{x}\right), a_{00}^{\prime}=0$. If $d_{y}^{\prime}$ is the largest $j$ such that $a_{0 j}^{\prime} \neq 0$, and since $a_{00}^{\prime}=0$ Theorem 3 implies that there are either $d_{y}^{\prime}$ (or infinitely many) linearly independent

$$
u_{i} \in \operatorname{ker} \mathbf{L}^{\prime} \cap \operatorname{ker} \mathbf{M}^{\prime}, \quad i=1, \ldots, d_{y}^{\prime} \quad(\text { or } i=1, \ldots, \infty) .
$$

Since $\mathbf{M}^{\prime}=D_{x}$, each of them is a function of the variable $y$ solely. Since $1 \in \operatorname{ker} \mathbf{L} \cap \operatorname{ker} \mathbf{L}$, we can choose (16) in such a way that $u_{1}=1$. Then

$$
\phi_{1}=\psi_{1}, \phi_{2}=\psi_{1} u_{2}, \ldots \in \operatorname{ker} \mathbf{L} \cap \operatorname{ker} \mathbf{M},
$$


and $\psi_{i} / \psi_{1}, i=1, \ldots, d_{y}^{\prime}$ are some function of $y$ solely. Thus, if the number $d_{y}^{\prime}$ is less then $k$, we cannot get $k$

$$
\psi_{1}, \psi_{2}, \ldots, \psi_{k} \in \operatorname{ker} \mathbf{L} \cap \operatorname{ker} \mathbf{M}
$$

Example 4 (the largest $j$ such that $a_{0 j} \neq 0$ is not invariant under gauge transformation). Consider the operator

$$
\mathbf{L}=D_{x}^{2} D_{y}+D_{y}^{2} D_{x}-\frac{1}{x} D_{y}^{2}+D_{y}
$$

and $\psi_{1}=x \in \operatorname{ker} \mathbf{L}$. Then

$$
\mathbf{L}^{\prime}=\mathbf{L}^{\psi_{1}}=D_{x}^{2} D_{y}+D_{y}^{2} D_{x}+\frac{2}{x} D_{x} D_{y}+D_{y} .
$$

Thus, $d_{y}=2$ and $d_{y}^{\prime}=1$, and there exists (a non-invertible) Darboux transformation generated by $\mathbf{M}=D_{x}-\psi_{1, x} \psi_{1}^{-1}=D_{x}-\frac{1}{x}$. It takes $\mathbf{L}$ into

$$
\mathbf{L}_{1}=D_{x}^{2} D_{y}+D_{y}^{2} D_{x}-\frac{1}{x} D_{y}^{2}+\left(1-\frac{2}{x^{2}}\right) D_{y},
$$

and the corresponding $\mathbf{N}$ is $\mathbf{N}=\mathbf{M}$.

Theorem 4 states some conditions under which an operator has non-invertible Darboux transformations generated by $\mathbf{M}$ in the form $D_{x}-\psi_{1, x} \psi_{1}^{-1}$ or $D_{y}-\psi_{1, y} \psi_{1}^{-1}$, where $\psi_{1} \in$ ker $\mathbf{L}$.

\section{Conclusions}

The paper provides the first ideas for the general (algebraic) theory of invertible first-order Darboux transformations for bivariate linear partial differential operators of arbitrary order $d$ and of arbitrary form. Although the order of the auxiliary operator has been restricted to the first order, the major operator is taken in general form.

Future work may include strengthening of Theorem 4 to describe a criteria. It is not known yet how to extend these ideas to the important three dimensional case, the generalized Laplace transformations for which have been recently developed in [3]. It is also extremely important to extend these ideas for discrete analogies that are under active development now $[4,7]$.

\section{References}

[1] Bagrov V.G., Samsonov B.F., Darboux transformation of the Schrödinger equation, Phys. Part. Nuclei 28 (1997), 374-397.

[2] Darboux G., Leçons sur la théorie générale des surfaces et les applications géométriques du calcul infinitésimal. II, Gauthier-Villars, Paris, 1889.

[3] Ganzha E.I., On Laplace and Dini transformations for multidimensional equations with a decomposable principal symbol, Program. Comput. Softw. 38 (2012), 150-155.

[4] Grinevich P.G., Novikov S.P., Discrete $S L_{2}$ connections and self-adjoint difference operators on the triangulated 2-manifold, arXiv:1207.1729.

[5] Li C.X., Nimmo J.J.C., Darboux transformations for a twisted derivation and quasideterminant solutions to the super KdV equation, Proc. R. Soc. Lond. Ser. A Math. Phys. Eng. Sci. 466 (2010), 2471-2493, arXiv:0911.1413.

[6] Matveev V.B., Salle M.A., Darboux transformations and solitons, Springer Series in Nonlinear Dynamics, Springer-Verlag, Berlin, 1991. 
[7] Novikov S.P., Four lectures: discretization and integrability. Discrete spectral symmetries, in Integrability, Lecture Notes in Physics, Vol. 767, Editor A.V. Mikhailov, Springer, Berlin, 2009, 119-138.

[8] Novikov S.P., Veselov A.P., Exactly solvable two-dimensional Schrödinger operators and Laplace transformations, in Solitons, geometry, and topology: on the crossroad, Amer. Math. Soc. Transl. Ser. 2, Vol. 179, Amer. Math. Soc., Providence, RI, 1997, 109-132, math-ph/0003008.

[9] Rogers C., Schief W.K., Bäcklund and Darboux transformations. Geometry and modern applications in soliton theory, Cambridge Texts in Applied Mathematics, Cambridge University Press, Cambridge, 2002.

[10] Shemyakova E., Laplace transformations as the only degenerate Darboux transformations of first order, Program. Comput. Softw. 38 (2012), 105-108.

[11] Shemyakova E., Proof of the completeness of Darboux Wronskian formulas for order two, Canad. J. Math., to appear, arXiv:1111.1338.

[12] Tsarev S.P., Factorization of linear partial differential operators and the Darboux method for integrating nonlinear partial differential equations, Theoret. Math. Phys. 122 (2000), 121-133.

[13] Tsarev S.P., Shemyakova E., Differential transformations of second-order parabolic operators in the plane, Proc. Steklov Inst. Math. 266 (2009), 219-227, arXiv:0811.1492. 